\title{
Identification of crystallographic planes of a polyhedral crystal at SENJU
}

\author{
A. Nakao', T. Moyoshi ${ }^{1}$, K. Moriyama', T. Matsumura², K. Iba ${ }^{2}$, S. Ohara ${ }^{3}$, Y. Ishikawa1, \\ K. Munakata ${ }^{1}$, T. Ohhara ${ }^{4}$, R. Kiyanagi ${ }^{4}$ \\ ${ }^{I}$ Neutron Science and Technology Center, CROSS, Tokai, Ibaraki 319-1106, Japan, \\ ${ }^{2}$ Department of Quantum Matter, AdSM, Hiroshima University, Hiroshima 739-8530, Japan, \\ ${ }^{3}$ Department of Physical Science and Engineering, Graduate School of Engineering, \\ Nagoya Institute of Technology, Nagoya 466-8555, Japan, \\ ${ }^{4}$ J-PARC Center, Japan Atomic Energy Agency, Tokai, Ibaraki 319-1195, Japan
}

$$
\text { a_nakao@corss.or.jp }
$$

SENJU of BL18 at MLF is a single-crystal neutron diffractometer with wide-area detectors, and it uses the time-of-flight Laue technique for structural analysis (Fig. 1) [1]. The integrated intensities measured for crystal structure and magnetic structure analyses are corrected for the Lorentz factor, incident spectrum and detector sensitivity difference. In addition, absorption correction is required for materials with large neutron absorption cross sections. Therefore, in order to perform numerical absorption correction based on the crystal shape, we developed a procedure to determine the Miller indices of polyhedral crystal faces using the photographs of crystals taken with a CCD camera and the UB matrix at SENJU [2]. When applied to DyNi3A19 [3], structural analysis demonstrated that the absorption effect could be corrected. Figure 2 shows a single crystal taken with a CCD camera, and the outline of the crystal is defined by the vertices.

In this presentation, we will report the details of the definition of crystal planes and the results of crystal structure analysis.

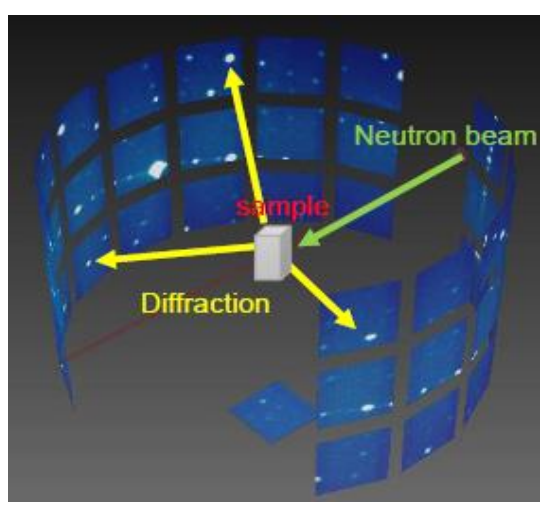

Figure 1. Single-crystal neutron Laue time-of-flight diffractometer SENJU

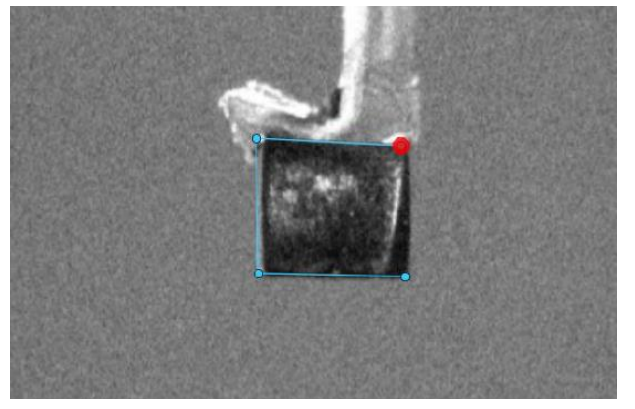

Figure 2. Single crystal captured by a CCD camera. The vertices of the crystal outline are connected by blue

[1] T. Ohhara, R. Kiyanagi, K. Oikawa, K. Kaneko,T. Kawasaki, I. Tamura, A. Nakao, T. Hanashima, K. Munakata, T. Moyoshi, T. Kuroda, H. Kimura,T. Sakakura, C. Lee, M. Takahashi, K. Ohshima, T. Kiyotani, Y. Noda \& M. Arai. (2016) J. Appl. Cryst. 49, 120.

[2] A. Nakao, T. Moyoshi, K. Moriyama, T. Matsumura, K. Iba, S. Ohara, Y. Ishikawa, K. Munakata, T. Ohhara, \& R. Kiyanagi. (2021) JPS Conf. Proc. 33011067

[3] K. Iba, T. Matsumura, A. Nakao, Y. Ishikawa, K. Ohishi, R. Kiyanagi, Y. Kousaka \& S. Ohara. (2020) JPS Conf. Proc. 30011164.

Keywords: single crystal, TOF Laue neutron diffraction, numerical absorption correction, Miller index

Improvements in the image-processing software and the STARGazer were developed in cooperation with Visible Information Center Inc. This work was partially supported by the Grant-in-Aid for Scientific Research (No. 19K03728) from the Ministry of Education, Culture, Sports, Science and Technology of Japan. The neutron diffraction measurement was performed on the approval of the Proposal Review Committee of J-PARC MLF (2018B106 and 201810018). 